\title{
Approximate Similarity Reduction for the Nonlinear $K(n, 1)$ Equation with Weak Damping via Symmetry Perturbation and Direct Method
}

\author{
Rui Zhang (Corresponding author) \\ Department of Mathematics, Northwest University \\ Chang'an district, Xi' an 710127, China
}

Tel: 86-29-8830-1750 E-mail: 1lz91@163.com

Lizi Lin

School of Economics and Management, Northwest University, Chang'an district, Xi' an 710127, China

Tel: 86-29-8640-2612Ｅ-mail: 1lz91@163.com

The research is financed by Asian Development Bank. No. 2006-A171(Sponsoring information)

\begin{abstract}
The nonlinear $K(n, 1)$ equation with weak damping is investigated via the approximate symmetry perturbation method and approximate direct method. The approximate symmetry and similarity reduction equations of different orders are derived and the corresponding series reduction solutions are obtained. As a result, the formal coincidence for both methods is displayed.
\end{abstract}

Keywords: Approximate symmetry perturbation method, Approximate direct method, Nonlinear $K(n, 1)$ equation

\section{Introduction}

In this paper, we intend to investigate the approximate similarity reductions and the infinite series reduction solutions for the nonlinear $K(n, 1)$ equation with weak damping (Biswas, 2009, p9-10)

$$
u_{t}+a\left(u^{n}\right)_{x}+u_{x x x}=-\epsilon u
$$

via the approximate symmetry perturbation (Zhao, Zhang and Lou, 2009, p1-4)(Jiao, Yao, Lou, 2008, p1-11 and the approximate direct methods (Clarkson and Kruskal, 1989, p2201-2212), where $\epsilon$ is a small parameter and $u$ is a function of $x$ and $t$. Hereafter, we put stress on the general case while $n>2$, irrespective of the simple case of $n=2$. In terms of the perturbation analysis (Cole, 1968)(Van Dyke, 1975)(Nayfeh, 2000) any solution to a perturbed PDE can be expressed as a series containing small parameter $\epsilon$

$$
u=\sum_{k=0}^{\infty} \epsilon^{k} u_{k}
$$

with $u_{k}$ functions of $x$ and $t$. Substituting Eq. (2) into Eq. (1) and vanishing the coefficients of all different powers of $\epsilon$, we obtain the following system

$$
u_{k, t}+n a \sum_{i_{1}+i_{2}+\cdots+i_{n}=k} u_{i_{1}} u_{i_{2}} \ldots u_{i_{(n-1)}} u_{i_{n}, x}+u_{k, x x x}=-u_{k-1},(k=0,1,2, \ldots)
$$

where $0 \leq i_{m} \leq k(m=1, \ldots, n)$ and $u_{-1}=0$.

In Sec. 2 and 3, we apply the approximate symmetry perturbation method and approximate direct method to Eq. (1) respectively. Sec. 4 shows the formal coincidence for both methods on the results obtained by both methods under certain transformations. The last section is the concluding remarks

\section{Approximate Symmetry Perturbation Method to Equation (1)}

In order to study Lie symmetry reduction of Eq. (3), we construct the Lie point symmetry in the vector form

$$
V=X \frac{\partial}{\partial x}+T \frac{\partial}{\partial t}+\sum_{k=0}^{\infty} U_{k} \frac{\partial}{\partial u_{k}}
$$

where $X, T$, and $U_{k}$ are functions of $x, t$, and $u_{k},(k=0,1, \ldots)$, equivalently, Eq. (3) is invariant under the transformation

$$
\left\{x, t, u_{k}, k=0,1, \ldots\right\} \rightarrow\left\{x+\varepsilon X, t+\varepsilon T, u_{k}+\varepsilon U_{k}, k=0,1, \ldots\right\},
$$


with infinitesimal parameter $\varepsilon$.

Since Eq. (1) is not explicitly dependent upon space-time $x, t$, the symmetry in the vector form (4) can be written as a function form

$$
\sigma_{k}=U_{k}-X U_{k, x}-T U_{k, t}, \quad(k=0,1, \ldots)
$$

Under notation (5), the symmetry equations for Eqs. (3) read

$$
\begin{aligned}
& \sigma_{k, t}+n a \sum_{i_{1}+i_{2}+\cdots+i_{n}=k}\left[\sigma_{i_{1}} u_{i_{2}} \ldots u_{i_{(n-2)}} u_{i_{(n-1)}} u_{i_{n}, x}+u_{i_{1}} \sigma_{i_{2}} \ldots u_{i_{(n-2)}} u_{i_{(n-1)}} u_{i_{n}, x}\right. \\
& \left.+\cdots+u_{i_{1}} u_{i_{2}} \ldots u_{i_{(n-2)}} u_{i_{(n-1)}} \sigma_{i_{n}, x}\right]+\sigma_{k, x x x}=-\sigma_{k-1},(k=0,1,2, \ldots)
\end{aligned}
$$

which are the linearized equations for Eqs. (3), with $0 \leq i_{m} \leq k(m=1, \ldots, n)$ and $\sigma_{-1}=0$.

It seems difficult to figure out $X, T$ and $U_{k},(k=0,1, \ldots)$ directly because there are infinite number of equations and arguments concerning or in $X, T$ and $U_{k},(k=0,1, \ldots)$. To make brief of it, we begin the discussion with finite number of equations.

Confining the range of $k$ to $(k=0-2)$ in Eqs. (3), (5) and (6), we see that $X, T, U_{0}, U_{1}$ and $U_{2}$ are functions of $x, t, u_{0}$, $u_{1}$ and $u_{2}$. In this case, the determining equations can be derived by substituting Eq. (5) into Eq. (6), eliminating $u_{0, t}, u_{1, t}$ and $u_{2, t}$ in terms of Eq. (3). Some of the determining equations read

$$
\begin{aligned}
& T_{x}=T_{u_{0}}=T_{u_{1}}=T_{u_{2}}=0, X_{t}=X_{u_{0}}=X_{u_{1}}=X_{u_{2}}=0, \\
& U_{0, u_{1}}=U_{0, u_{2}}=U_{0, u_{0} u_{0}}=0, U_{1, u_{0}}=U_{1, u_{2}}=U_{1, u_{1} u_{1}}=0, \\
& U_{2, u_{0}}=U_{2, u_{1}}=U_{2, u_{2} u_{2}}=0 .
\end{aligned}
$$

The general solution to Eqs. (7) is

$$
\begin{aligned}
& X=X(x), T=T(t), U_{0}=a_{1}(x, t) u_{0}+a_{0}(x, t), \\
& U_{1}=a_{3}(x, t) u_{1}+a_{2}(x, t), U_{2}=a_{5}(x, t) u_{2}+a_{4}(x, t) .
\end{aligned}
$$

Using relations (8), the remaining determining equations are immediately simplified to

$$
\begin{aligned}
& a_{1}=-\frac{2}{n-1} X_{x}=\frac{1}{n-1}\left(X_{x}-T_{t}\right), a_{3}+(n-2) a_{1}=X_{x}, \\
& a_{5}+(n-1) a_{1}-a_{3}=X_{x}, a_{0}=0, a_{2}=0, a_{4}=0, X_{x x}=0 .
\end{aligned}
$$

It is straightforward to find that

$$
\begin{aligned}
& X=\frac{c}{3} x+x_{0}, T=c t+t_{0}, U_{0}=-\frac{2}{3(n-1)} c u_{0}, \\
& U_{1}=\left(1-\frac{2}{3(n-1)}\right) c u_{1}, U_{2}=\left(2-\frac{2}{3(n-1)}\right) c u_{2} .
\end{aligned}
$$

Likewise, restricting the range of $k$ to $\{k \mid k=0,1,2,3\}$ in Eqs. (3) (5) and (6), where $X, T, U_{0}, U_{1}, U_{2}$ and $U_{3}$ are functions of $x, t, u_{0}, u_{1}, u_{2}$ and $u_{3}$, repeating the calculation process as before, then we have

$$
\begin{aligned}
& X=\frac{c}{3} x+x_{0}, T=c t+t_{0}, \\
& U_{0}=-\frac{2}{3(n-1)} c u_{0}, U_{1}=\left(1-\frac{2}{3(n-1)}\right) c u_{1}, \\
& U_{2}=\left(2-\frac{2}{3(n-1)}\right) c u_{2}, U_{3}=\left(3-\frac{2}{3(n-1)}\right) c u_{3} .
\end{aligned}
$$

With more similar computation considered, we find that $X, T$ and $U_{k}(k=0,1, \ldots)$ are formally coherent, i.e.,

$$
X=\frac{c}{3} x+x_{0}, T=c t+t_{0}, U_{k}=\left(k-\frac{2}{3(n-1)}\right) c u_{k},(k=0,1, \ldots)
$$

where $c, x_{0}$ and $t_{0}$ are arbitrary constants.

Subsequently, solving the characteristic equations

$$
\frac{d x}{X}=\frac{d t}{T}, \frac{d u_{0}}{U_{0}}=\frac{d t}{T}, \ldots, \frac{d u_{k}}{U_{k}}=\frac{d t}{T}, \ldots
$$


leads to the similarity solutions to Eq. (3). Two subcases are distinguished as follows.

Case 1: When $c \neq 0$, without loss of generality, making the transformation $x_{0} \longrightarrow \frac{1}{3} c x_{0}$ and $t_{0} \longrightarrow c t_{0}$, we rewrite Eq. (9) as

$$
\begin{aligned}
& X=\frac{1}{3} c\left(x+x_{0}\right), T=c\left(t+t_{0}\right), U_{0}=-\frac{2}{3(n-1)} c u_{0}, \\
& U_{1}=\left(1-\frac{2}{3(n-1)}\right) c u_{1}, \ldots, U_{k}=\left(k-\frac{2}{3(n-1)}\right) c u_{k},(k=0,1, \ldots)
\end{aligned}
$$

in this case, solving Eq. (10) leads to the following invariants

$$
\begin{gathered}
I(x, t)=\xi=\left(x+x_{0}\right)\left(t+t_{0}\right)^{-\frac{1}{3}}, \\
I_{0}(x, t)=V_{0}=\left(t+t_{0}\right)^{\frac{2}{3(n-1)}} u_{0},
\end{gathered}
$$

and

$$
I_{k}(x, t)=V_{k}=\left(t+t_{0}\right)^{\frac{2}{3(n-1)}-k} u_{k},(k=1,2, \ldots)
$$

viewing $V_{k}(k=0,1, \ldots)$ as functions of $\xi$, we get the similarity solutions

$$
u_{k}=V_{k}(\xi)\left(t+t_{0}\right)^{k-\frac{2}{3(n-1)}},(k=0,1, \ldots)
$$

to Eqs. (3) with similarity variable

$$
\xi=\left(x+x_{0}\right)\left(t+t_{0}\right)^{-\frac{1}{3}}
$$

From Eq. (2), the series reduction solution to Eq. (1) is given by

$$
u=\sum_{k=0}^{\infty} \epsilon^{k}\left(t+t_{0}\right)^{k-\frac{2}{3(n-1)}} V_{k}(\xi),(k=0,1, \ldots)
$$

substituting Eqs. (12) into Eqs. (3), we get the following related similarity reduction equations

$$
\begin{array}{ll}
O\left(\epsilon^{0}\right): \quad & V_{0, \xi \xi \xi}+n a V_{0}^{n-1} V_{0, \xi}-\frac{2}{3(n-1)} V_{0}-\frac{1}{3} \xi V_{0, \xi}=0, \\
O\left(\epsilon^{1}\right): \quad & V_{1, \xi \xi \xi}+n a V_{0}^{n-1} V_{1, \xi}+n(n-1) a V_{0}^{n-2} V_{1} V_{0, \xi} \\
& +\left(1-\frac{2}{3(n-1)}\right) V_{1}-\frac{1}{3} \xi V_{1, \xi}=-V_{0}, \\
O\left(\epsilon^{2}\right): \quad & V_{2, \xi \xi \xi}+n a V_{0}^{n-1} V_{2, \xi}+n(n-1) a V_{0}^{n-2} V_{1} V_{1, \xi}+n(n-1) a V_{0}^{n-2} V_{2} V_{0, \xi} \\
& +\frac{n(n-1)(n-2)}{2} a V_{0}^{n-3} V_{1}^{2} V_{0, \xi}+\left(2-\frac{2}{3(n-1)}\right) V_{2}-\frac{1}{3} \xi V_{2, \xi}=-V_{1}, \\
& \left.\ldots, \quad V_{k, \xi \xi \xi}+n a \quad \sum_{i_{1}}^{k}\right): \quad V_{i_{1}} V_{i_{2}} \ldots V_{i_{(n-1)}} V_{i_{n}, \xi} \\
& +\left(k-\frac{2}{3(n-1)}\right) V_{k}-\frac{1}{3} \xi V_{k, \xi}=-V_{k-1},
\end{array}
$$

with $0 \leq i_{m} \leq k,(m=1, \ldots, n)$ and $V_{-1}=0$. The $k$ th $(k>0)$ similarity reduction equation is in fact a third order linear ordinary differential equation (ODE) of $V_{k}$ when the previous $V_{0}, V_{1}, \ldots, V_{k-1}$ are known, since it can be rewritten as

$$
\begin{aligned}
& V_{k, \xi \xi \xi}+n a\left[V_{0}{ }^{n-1} V_{k, \xi}+(n-1) V_{0}{ }^{n-2} V_{k} V_{0, \xi}\right] \\
& +\left(k-\frac{2}{3(n-1)}\right) V_{k}-\frac{1}{3} \xi V_{k, \xi}=G_{k}(\xi),(k=0,1, \ldots)
\end{aligned}
$$

where $G_{k}$ is an only function of $\left\{V_{0}, V_{1}, \ldots V_{k-1}\right\}$

$$
G_{k}(\xi)=-V_{k-1}-n a \sum_{i_{1}+i_{2}+\cdots+i_{n}=k} V_{i_{1}} V_{i_{2}} \ldots V_{i_{(n-1)}} V_{i_{n}, \xi}, \quad(k=0,1, \ldots)
$$

with $i_{m} \neq k(m=1, \ldots, n)$.

Case 2: When $c=0$, we have

$$
X=x_{0}, T=t_{0}, U_{k}=\left(k-\frac{2}{3(n-2)}\right) c u_{k}=0,(k=1,2, \ldots)
$$


the similarity solutions are

$$
u_{k}=V_{k}(\xi), \xi=t_{0} x-x_{0} t,(k=1,2, \ldots, n)
$$

thus the series reduction solution to Eq. (1) is

$$
u=\sum_{k=0}^{\infty} \epsilon^{k} V_{k}(\xi)
$$

where $V_{k}(\xi)(k=0,1,2 \ldots)$ yields

$$
\begin{array}{ll}
O\left(\epsilon^{0}\right): & \left(t_{0}\right)^{3} V_{0, \xi \xi \xi}+n a t_{0} V_{0}^{n-1} V_{0, \xi}-x_{0} V_{0, \xi}=0, \\
O\left(\epsilon^{1}\right): & \left(t_{0}\right)^{3} V_{1, \xi \xi \xi}+n a t_{0} V_{0}^{n-1} V_{1, \xi}+n(n-1) a t_{0} V_{0}^{n-2} V_{1} V_{0, \xi}-x_{0} V_{1, \xi}=-V_{0}, \\
& \ldots, \\
O\left(\epsilon^{k}\right): \quad & \left(t_{0}\right)^{3} V_{k, \xi \xi \xi}+n a t_{0} \sum_{i_{1}+i_{2}+\cdots+i_{n}=k} V_{i_{1}} V_{i_{2}} \ldots V_{i_{(n-1)}} V_{i_{n}, \xi}-x_{0} V_{k, \xi}=-V_{k-1},
\end{array}
$$

with $0 \leq i_{m} \leq k,(m=1, \ldots, n)$ and $V_{-1}=0$. The $k$ th $(k>0)$ similarity reduction equation can be rewritten as an ODE

$$
\left(t_{0}\right)^{3} V_{k, \xi \xi \xi}+n a t_{0}\left[V_{0}{ }^{n-1} V_{k, \xi}+(n-1) V_{0}{ }^{n-2} V_{k} V_{0, \xi}\right]-x_{0} V_{k, \xi}=G_{k}(\xi),(k=0,1, \ldots)
$$

of $V_{k}(\xi)$, where $G_{k}$ is a function of $\left\{V_{0}, V_{1}, \ldots, V_{k-1}\right\}$ defined as

$$
G_{k}(\xi)=-V_{k-1}-n a t_{0} \sum_{i_{1}+i_{2}+\cdots+i_{n}=k} V_{i_{1}} V_{i_{2}} \ldots V_{i_{(n-1)}} V_{i_{n}, \xi},(k=0,1, \ldots)
$$

with $i_{m} \neq k(m=1, \ldots, n)$.

\section{Approximate Direct Method to Equation (1)}

In this section, we develop the direct method to investigate Eq. (3) for its similarity solutions of the form

$$
u_{k}=f_{k}\left(x, t, P_{k}(z(x, t))\right),(k=0,1, \ldots)
$$

which satisfy a system of ODEs resulting from inserting Eq. (16) into Eq. (3).

On substituting Eq. (16) into Eq. (3), since only one term $u_{k, x x x}$ in Eq. (3) generates the terms $P_{k, z z z}$ and $P_{k, z} P_{k, z z}$ during the substitution, it is easily seen that the coefficients of $P_{k, z z z}$ and $P_{k, z} P_{k, z z}$ are $f_{k, P_{k}}\left(z_{x}\right)^{3}$ and $3 f_{k, P_{k} P_{k}}\left(z_{x}\right)^{3}$, respectively. We reserve uppercase Greek letters for undetermined functions of $z$ hereafter. The ratios of the coefficients are functions of $z$, namely,

$$
f_{k, P_{k}}\left(z_{x}\right)^{3}=3 f_{k, P_{k} P_{k}}\left(z_{x}\right)^{3} \Gamma_{k}(z),(k=0,1, \ldots)
$$

with the solution

$$
f_{k}=\alpha_{k}(x, t)+\beta_{k}(x, t) e^{\frac{1}{3 \Gamma(2)} P_{k}},(k=0,1, \ldots)
$$

where $\alpha_{k}(x, t)$ and $\beta_{k}(x, t)$ are arbitrary functions. Hence, rewriting $e^{\frac{1}{3(x)} P_{k}}$ as $P_{k}$, it is sufficient to seek the similarity reduction of Eq. (3) in the special form

$$
u_{k}=\alpha_{k}(x, t)+\beta_{k}(x, t) P_{k}(z(x, t)),(k=0,1, \ldots)
$$

instead of the general form Eq. (16).

Remark: Three freedoms in the determination of $\alpha_{k}(x, t), \beta_{k}(x, t)$ and $z(x, t)$ should be notified:

(i) If $\alpha_{k}(x, t)=\alpha_{k}^{\prime}(x, t)+\beta_{k}(x, t) \Omega(z)$, then one can take $\Omega(z)=0$;

(ii) If $\beta_{k}(x, t)=\beta_{k}^{\prime}(x, t) \Omega(z)$, then one can take $\Omega(z)=$ constant;

(iii) If $z(x, t)$ is determined by $\Omega(z)=z_{0}(x, t)$, where $\Omega(z)$ is any invertible function, then one can take $\Omega(z)=z$.

Substituting Eq. (17) into Eq. (3), we find that the coefficients for $P_{0, z z z}, P_{0}^{n-1} P_{0, z}, P_{0, z z}$ and $P_{0}^{n-2} P_{0, z}$ are $\beta_{0}\left(z_{x}\right)^{3}, n a \beta_{0}^{n} z_{x}$, $3 \beta_{0, x}\left(z_{x}\right)^{2}+3 \beta_{0} z_{x} z_{x x}$ and $n(n-1) a \alpha_{0} \beta_{0}^{n-1} z_{x}$, respectively. Since $P_{k}$ is only a function of $z$, it requires that

$$
\begin{aligned}
n a \beta_{0}^{n} z_{x} & =\beta_{0}\left(z_{x}\right)^{3} \Phi_{0}(z), \\
3 \beta_{0, x}\left(z_{x}\right)^{2}+3 \beta_{0} z_{x} z_{x x} & =\beta_{0}\left(z_{x}\right)^{3} \Psi_{0}(z), \\
n(n-1) a \alpha_{0} \beta_{0}^{n-1} z_{x} & =\beta_{0}\left(z_{x}\right)^{3} \Omega_{0}(z) .
\end{aligned}
$$

From Eq. (18) and remark (ii), we get

$$
\beta_{0}=z_{x}^{\frac{2}{n-1}}
$$


From Eq. (20) and remark (i), we can see $\alpha_{0}=0$.

From Eqs. (19), (21) and remark (iii), we have

$$
\frac{6}{n-1} z_{x} z_{x x}+3 z_{x} z_{x x}=z_{x}^{3} \Psi_{0}(z)
$$

then

$$
z=\theta(t) x+\sigma(t)
$$

where $\theta(t)$ and $\sigma(t)$ are some functions to be settled.

Then Eq. (3) is degenerated into

$$
\theta^{4} P_{0, z z z}+n a \theta^{4} P_{0}^{n-1} P_{0, z}+\theta\left(x \theta_{t}+\sigma_{t}\right) P_{0, z}+\frac{2}{n-1} \theta_{t} P_{0}=0 .
$$

From the coefficients of $P_{0, z z z}, P_{0, z}$ and $P_{0}$ and the relations

$$
x \theta_{t}+\sigma_{t}=\theta^{3} \Gamma_{1}(z), \frac{2}{n-1} \theta_{t}=\theta^{4} \Gamma_{2}(z),
$$

we have

$$
\Gamma_{1}(z)=A z+B, \quad \Gamma_{2}(z)=\frac{2}{n-1} A, \quad \frac{d \theta}{d t}=A \theta^{4}, \quad \frac{d \sigma}{d t}=\theta^{3}(A \sigma+B),
$$

where $A$ and $B$ are arbitrary constants.

Assume that $k \geq 1$, inserting Eq. (17) into Eq. (3), we know that the coefficients of $P_{k-1}, P_{0}^{n-2} P_{0, z}$ and $P_{k, z z z}$ are $-\beta_{k-1}$, $n(n-1) a \beta_{0}^{n-1} z_{x} \alpha_{k}$ and $\beta_{k} z_{x}^{3}$ respectively, which leads to

$$
-\beta_{k-1}=\beta_{k} z_{x}^{3} \Phi_{k}(z), \quad n(n-1) a \beta_{0}^{n-1} z_{x} \alpha_{k}=\beta_{k} z_{x}^{3} \Psi_{k}(z), \quad(k \geq 1)
$$

then using remark (i) and (ii), we have

$$
\alpha_{k}=0, \beta_{k}=\left(z_{x}\right)^{\frac{2}{n-1}-3 k}(k=0,1,2, \ldots) .
$$

We distinguish the following two subcases.

Case 1: When $A \neq 0$, Eq. (23) has solution

$$
\theta=-\left(3 A\left(t-t_{0}\right)\right)^{-\frac{1}{3}}, \quad \sigma=-\frac{B}{A}+s_{0}\left(t-t_{0}\right)^{-\frac{1}{3}},
$$

where $t_{0}$ and $s_{0}$ are arbitrary constants.

In terms of Eqs. (17), (21), (22), (23) and (24), we get the following solution to Eq. (3)

$$
u_{k}=(-1)^{k}\left(3 A\left(t-t_{0}\right)\right)^{k-\frac{2}{3(n-1)}} P_{k}(z),(k=0,1,2, \ldots)
$$

where the similarity variable $z=-\left(3 A\left(t-t_{0}\right)\right)^{-\frac{1}{3}} x+s_{0}\left(t-t_{0}\right)^{-\frac{1}{3}}-\frac{B}{A}$.

From Eqs. (25) and (2), we obtain the series reduction solution

$$
u=\sum_{k=0}^{\infty}(-1)^{k} \epsilon^{k}\left(3 A\left(t-t_{0}\right)\right)^{k-\frac{2}{3(n-1)}} P_{k}(z),(k=0,1,2, \ldots)
$$

to Eq. (1). Inserting Eq. (25) into Eq. (3), we get the similarity reduction equations

$$
\begin{aligned}
& P_{k, z z z}+n a \sum_{i_{1}+i_{2}+\cdots+i_{n}=k} P_{i_{1}} P_{i_{2}} \ldots P_{i_{(n-1)}} P_{i_{n}, z}+(A z+B) P_{k, z} \\
& +\left(\frac{2}{n-1}-3 k\right) A P_{k}=-P_{k-1},(k=0,1,2, \ldots)
\end{aligned}
$$

with $P_{-1}=0$.

Case 2: When $A=0$, Eq. (23) has the solution

$$
\theta=t_{0}, \quad \sigma=B t_{0}^{3} t+s_{0}
$$


where $t_{0}$ and $s_{0}$ are arbitrary constants. By Eqs. (17), (21), (22), (23) and (29), we obtain the similarity solution

$$
u_{k}=t_{0}^{\frac{2}{n-1}-3 k} P_{k}(z),(k=0,1,2, \ldots)
$$

with the similarity variable $z=t_{0} x+B t_{0}^{3} t+s_{0}$. Based on this, the series reduction solution to Eq. (1) is

$$
u=\sum_{k=0}^{\infty} \epsilon^{k} t_{0}^{\frac{2}{n-1}-3 k} P_{k}(z)
$$

and the similarity reduction equation is boiled down to

$$
P_{k, z z z}+n a \sum_{i_{1}+i_{2}+\cdots+i_{n}=k} P_{i_{1}} P_{i_{2}} \ldots P_{i_{(n-1)}} P_{i_{n}, z}+B P_{k, z}=-P_{k-1},(k=0,1,2, \ldots)
$$

with $P_{-1}=0$.

\section{Analysis on Formal Coincidence for Both Methods}

In the following, we discuss the formal coincidence for both methods on the basis of the results obtained by both methods.

Case 1: We now compare Eqs. (25) and (26) with the results concerning similarity reduction equations and similarity solutions in Case 1 of Sec. 2. By the transformations $A \rightarrow-\frac{1}{3}, B \rightarrow 0, t_{0} \rightarrow-t_{0}$ and $s_{0} \rightarrow x_{0}$, we can get the similarity variable $z=\left(x+x_{0}\right)\left(t+t_{0}\right)^{-\frac{1}{3}}$, then Eqs. (25) and (26) are respectively changed into

$$
u_{k}=\left(t+t_{0}\right)^{k-\frac{2}{3(n-1)}} P_{k}(z),(k=0,1,2, \ldots)
$$

and

$$
\begin{aligned}
& P_{k, z z z}+n a \sum_{i_{1}+i_{2}+\cdots+i_{n}=k} P_{i_{1}} P_{i_{2}} \ldots P_{i_{(n-1)}} P_{i_{n}, z}-\frac{1}{3} z P_{k, z} \\
& +\left(k-\frac{2}{3(n-1)}\right) P_{k}=-P_{k-1}, \quad(k=0,1,2, \ldots),
\end{aligned}
$$

with $P_{-1}=0$.

On the other hand, for Case 1 in Sec. 2, making the transformation $V_{k}(\xi) \rightarrow P_{k}(\xi)$, Eqs. (12) and (15) are respectively converted into

$$
u_{k}=\left(t+t_{0}\right)^{k-\frac{2}{3(n-1)}} P_{k}(\xi),(k=0,1,2, \ldots)
$$

and

$$
\begin{aligned}
& P_{k, \xi \xi \xi}+n a \sum_{i_{1}+i_{2}+\cdots+i_{n}=k} P_{i_{1}} P_{i_{2}} \ldots P_{i_{(n-1)}} P_{i_{n}, \xi}-\frac{1}{3} \xi P_{k, \xi} \\
& +\left(k-\frac{2}{3(n-1)}\right) P_{k}=-P_{k-1}, \quad(k=0,1,2, \ldots)
\end{aligned}
$$

where $P_{-1}=0$, which are formally the same as Eqs. (27) and (28).

Case 2: Suppose that $t_{0} \neq 0$, by the transformations $B \rightarrow-\frac{x_{0}}{t_{0}^{3}}, t_{0} \rightarrow t_{0}$ and $s_{0} \rightarrow 0$, Eqs. (30) and (31) are respectively transformed into

$$
u_{k}=t_{0}^{\frac{2}{n-1}-3 k} P_{k}(z),(k=0,1,2, \ldots)
$$

and

$$
u=\sum_{k=0}^{\infty} \epsilon^{k} t_{0}^{\frac{2}{n-1}-3 k} P_{k}(z)
$$

with similarity variable $z=t_{0} x-x_{0} t$, then Eq. (32) becomes

$$
P_{k, z z z}+n a \sum_{i_{1}+i_{2}+\cdots+i_{n}=k} P_{i_{1}} P_{i_{2}} \ldots P_{i_{(n-1)}} P_{i_{n}, z}-\frac{x_{0}}{t_{0}^{3}} P_{k, z}=-P_{k-1}, \quad(k=0,1,2, \ldots)
$$


with $P_{-1}=0$.

Meanwhile, for Case 2 in Sec. 2, $V_{k}(\xi) \rightarrow t_{0}^{\frac{2}{n-1}-3 k} P_{k}(\xi)$ maps Eqs. (23) and (25) into

$$
u_{k}=t_{0}^{\frac{2}{n-1}-3 k} P_{k}(\xi),(k=0,1,2, \ldots)
$$

and

$$
P_{k, \xi \xi \xi}+n a \sum_{i_{1}+i_{2}+\cdots+i_{n}=k} P_{i_{1}} P_{i_{2}} \ldots P_{i_{(n-1)}} P_{i_{n}, \xi}-\frac{x_{0}}{t_{0}^{3}} P_{k, \xi}=-P_{k-1},(k=0,1,2, \ldots)
$$

with $P_{-1}=0$, which are formally equivalent to Eqs. (49) and (34) respectively.

From the above analysis of the results from both methods, we can see that approximate direct method produces more general approximate similarity reduction than the approximate symmetry perturbation method does.

\section{Conclusion}

To sum up, applying the approximate symmetry perturbation method and the approximate direct method to the nonlinear $K(n, 1)$ equation with weak damping, we have summarized the similarity reduction equations of different orders in uniform forms and obtained the infinite series similarity reduction solutions in general formulas for Eq. (1). As a result, we have demonstrated the formal coincidence for both methods by relating both results. It is interesting to take both methods into account while dealing with other perturbed PDEs. Moreover, the extension of approximate Lie symmetry perturbation method to approximate nonclassical symmetry ones is likely to improve the method.

\section{Acknowledgment}

The authors are grateful to Shunli Zhang and Lizi Lin, whose comments and suggestions of the earlier versions of the paper have led to a substantial clarification and revision of work.

\section{References}

A. Biswas. (2009). Solitary wave solution for the generalized KdV equation with time-dependent damping and dispersion. Communications in Nonlinear Science and Numerical Simulation, 14(9-10), 3503-3506.

A. H. Nayfeh. (2000). Perturbation Methods, John Wiley and Sons, New York.

J. D. Cole. (1968). Perturbation Methods in Applied Mathematics, Blaisdell Publishing Company, Walthma Massachusetts.

M. Van Dyke. (1975). Perturbation Methods in Fluid Mechanics, CA: Parabolic Press, Stanford.

P. A. Clarkson and M. D. Kruskal. (1989). New similarity reductions of the Boussinesq equation. Journal of Mathematical Physics, 30, 2201-2212.

X. Y. Jiao, R. X. Yao \& S. Y. Lou. (2008). Approximate similarity reduction for singularly perturbed Boussinesq equation via symmetry perturbation and direct method. Journal of Mathematical Physics, 49, 093505.

Y. Zhao, S. L. Zhang and S. Y. Lou. (2009). Approximate Symmetry Reduction and Infinite Series Solutions to the Nonlinear Wave Equation with Damping.Chinese Physics Letter, 26, 100201. 